\title{
Classifying Idiomatic and Literal Expressions Using Topic Models and Intensity of Emotions
}

\author{
Jing Peng \& Anna Feldman \\ Computer Science/Linguistics \\ Montclair State University \\ Montclair, New Jersey, USA \\ Ekaterina Vylomova \\ Computer Science \\ Bauman State Technical University \\ Moscow, Russia \\ \{pengj, feldmana\}@mail.montclair.edu evylomova@gmail.com
}

\begin{abstract}
We describe an algorithm for automatic classification of idiomatic and literal expressions. Our starting point is that words in a given text segment, such as a paragraph, that are highranking representatives of a common topic of discussion are less likely to be a part of an idiomatic expression. Our additional hypothesis is that contexts in which idioms occur, typically, are more affective and therefore, we incorporate a simple analysis of the intensity of the emotions expressed by the contexts. We investigate the bag of words topic representation of one to three paragraphs containing an expression that should be classified as idiomatic or literal (a target phrase). We extract topics from paragraphs containing idioms and from paragraphs containing literals using an unsupervised clustering method, Latent Dirichlet Allocation (LDA) (Blei et al., 2003). Since idiomatic expressions exhibit the property of non-compositionality, we assume that they usually present different semantics than the words used in the local topic. We treat idioms as semantic outliers, and the identification of a semantic shift as outlier detection. Thus, this topic representation allows us to differentiate idioms from literals using local semantic contexts. Our results are encouraging.
\end{abstract}

\section{Introduction}

The definition of what is literal and figurative is still object of debate. Ariel (2002) demonstrates that literal and non-literal meanings cannot always be distinguished from each other. Literal meaning is originally assumed to be conventional, compositional, relatively context independent, and truth conditional. The problem is that the boundary is not clear-cut, some figurative expressions are compositional - metaphors and many idioms; others are conventional - most of the idioms. Idioms present great challenges for many Natural Language Processing (NLP) applications. They can violate selection restrictions (Sporleder and $\mathrm{Li}, 2009$ ) as in push one's luck under the assumption that only concrete things can normally be pushed. Idioms can disobey typical subcategorization constraints (e.g., in line without a determiner before line), or change the default assignments of semantic roles to syntactic categories (e.g., in $X$ breaks something with $Y$, $\mathrm{Y}$ typically is an instrument but for the idiom break the ice, it is more likely to fill a patient role as in How to break the ice with a stranger). In addition, many potentially idiomatic expressions can be used either literally or figuratively, depending on the context. This presents a great challenge for machine translation. For example, a machine translation system must translate held fire differently in Now, now, hold your fire until I've had a chance to explain. Hold your fire, Bill. You're too quick to complain. and The sergeant told the soldiers to hold their fire. Please hold your fire until I get out of the way. In fact, we tested the last two examples using the Google Translate engine and we got proper translations of the two neither into Russian nor into Hebrew, Spanish, or Chinese. Most current translation systems rely on large repositories of idioms. Unfortunately, these systems are not capable to tell apart literal from figurative usage of the same expression in context. Despite the common perception that phrases that can be idioms are mainly used in their idiomatic sense, Fazly et al. (2009)'s analysis of 60 idioms has shown that close to half of these also have a clear literal meaning; and of those with a literal meaning, on average around $40 \%$ of their usages are literal.

In this paper we describe an algorithm for automatic classification of idiomatic and literal expressions. Our starting point is that words in a given text segment, such as a paragraph, that are high-ranking representatives of a common topic of discussion are less likely to be a part of an idiomatic expression. Our additional hypothesis is that contexts in which idioms occur, typically, are more affective and therefore, we incorporate a simple analysis of the intensity of the emotions expressed by the contexts. We investigate the bag of words topic representation of one to three paragraphs containing an expression that should be classified as idiomatic or literal (a target phrase). We extract topics from paragraphs containing idioms and from paragraphs containing literals using an unsupervised clustering method, Latent Dirichlet Allocation (LDA) (Blei et al., 2003). Since idiomatic expressions exhibit the property of non-compositionality, we assume that they usually present different semantics than the words used 
in the local topic. We treat idioms as semantic outliers, and the identification of semantic shift as outlier detection. Thus, this topic representation allows us to differentiate idioms from literals using the local semantics.

The paper is organized as follows. Section 2 briefly describes previous approaches to idiom recognition or classification. In Section 3 we describe our approach in detail, including the hypothesis, the topic space representation, and the proposed algorithm. After describing the preprocessing procedure in Section 4 , we turn to the actual experiments in Sections 5 and 6. We then compare our approach to other approaches (Section 7) and discuss the results (Section 8).

\section{Previous Work}

Previous approaches to idiom detection can be classified into two groups: 1) Type-based extraction, i.e., detecting idioms at the type level; 2) token-based detection, i.e., detecting idioms in context. Type-based extraction is based on the idea that idiomatic expressions exhibit certain linguistic properties that can distinguish them from literal expressions (Sag et al. (2002); Fazly et al. (2009)), among many others, discuss various properties of idioms. Some examples of such properties include 1) lexical fixedness: e.g., neither 'shoot the wind' nor 'hit the breeze' are valid variations of the idiom shoot the breeze and 2) syntactic fixedness: e.g., The guy kicked the bucket is potentially idiomatic whereas The bucket was kicked is not idiomatic anymore; and of course, 3) non-compositionality. Thus, some approaches look at the tendency for words to occur in one particular order, or a fixed pattern. Hearst (1992) identifies lexico-syntactic patterns that occur frequently, are recognizable with little or no precoded knowledge, and indicate the lexical relation of interest. Widdows and Dorow (2005) use Hearst's concept of lexicosyntactic patterns to extract idioms that consist of fixed patterns between two nouns. Basically, their technique works by finding patterns such as "thrills and spills", whose reversals (such as "spills and thrills") are never encountered.

While many idioms do have these properties, many idioms fall on the continuum from being compositional to being partly unanalyzable to completely noncompositional (Cook et al. (2007)). Fazly et al. (2009); $\mathrm{Li}$ and Sporleder (2010), among others, notice that type-based approaches do not work on expressions that can be interpreted idiomatically or literally depending on the context and thus, an approach that considers tokens in context is more appropriate for the task of idiom recognition.

A number of token-based approaches have been discussed in the literature, both supervised (Katz and Giesbrech (2006)), weakly supervised (Birke and Sarkar (2006)) and unsupervised (Sporleder and $\mathrm{Li}$ (2009); Fazly et al. (2009)). Fazly et al. (2009) develop statistical measures for each linguistic property of idiomatic expressions and use them both in a type- based classification task and in a token identification task, in which they distinguish idiomatic and literal usages of potentially idiomatic expressions in context. Sporleder and Li (2009) present a graph-based model for representing the lexical cohesion of a discourse. Nodes represent tokens in the discourse, which are connected by edges whose value is determined by a semantic relatedness function. They experiment with two different approaches to semantic relatedness: 1) Dependency vectors, as described in Pado and Lapata (2007); 2) Normalized Google Distance (Cilibrasi and Vitányi (2007)). Sporleder and Li (2009) show that this method works better for larger contexts (greater than five paragraphs). Li and Sporleder (2010) assume that literal and figurative data are generated by two different Gaussians, literal and non-literal and the detection is done by comparing which Gaussian model has a higher probability to generate a specific instance. The approach assumes that the target expressions are already known and the goal is to determine whether this expression is literal or figurative in a particular context. The important insight of this method is that figurative language in general exhibits less semantic cohesive ties with the context than literal language.

Feldman and Peng (2013) describe several approaches to automatic idiom identification. One of them is idiom recognition as outlier detection. They apply principal component analysis for outlier detection - an approach that does not rely on costly annotated training data and is not limited to a specific type of a syntactic construction, and is generally language independent. The quantitative analysis provided in their work shows that the outlier detection algorithm performs better and seems promising. The qualitative analysis also shows that their algorithm has to incorporate several important properties of the idioms: (1) Idioms are relatively non-compositional, comparing to literal expressions or other types of collocations. (2) Idioms violate local cohesive ties, as a result, they are semantically distant from the local topics. (3) While not all semantic outliers are idioms, non-compositional semantic outliers are likely to be idiomatic. (4) Idiomaticity is not a binary property. Idioms fall on the continuum from being compositional to being partly unanalyzable to completely non-compositional.

The approach described below is taking Feldman and Peng (2013)'s original idea and is trying to address (2) directly and (1) indirectly. Our approach is also somewhat similar to Li and Sporleder (2010) because it also relies on a list of potentially idiomatic expressions.

\section{Our Hypothesis}

Similarly to Feldman and Peng (2013), out starting point is that idioms are semantic outliers that violate cohesive structure, especially in local contexts. However, our task is framed as supervised classification and we rely on data annotated for idiomatic and literal expressions. We hypothesize that words in a given text 
segment, such as a paragraph, that are high-ranking representatives of a common topic of discussion are less likely to be a part of an idiomatic expression in the document.

\subsection{Topic Space Representation}

Instead of the simple bag of words representation of a target document (segment of three paragraphs that contains a target phrase), we investigate the bag of words topic representation for target documents. That is, we extract topics from paragraphs containing idioms and from paragraphs containing literals using an unsupervised clustering method, Latent Dirichlet Allocation (LDA) (Blei et al., 2003). The idea is that if the LDA model is able to capture the semantics of a target document, an idiomatic phrase will be a "semantic" outlier of the themes. Thus, this topic representation will allow us to differentiate idioms from literals using the semantics of the local context.

Let $d=\left\{w_{1}, \cdots, w_{N}\right\}^{t}$ be a segment (document) containing a target phrase, where $N$ denotes the number of terms in a given corpus, and $t$ represents transpose. We first compute a set of $m$ topics from $d$. We denote this set by

$$
T(d)=\left\{t_{1}, \cdots, t_{m}\right\}
$$

where $t_{i}=\left(w_{1}, \cdots, w_{k}\right)^{t}$. Here $w_{j}$ represents a word from a vocabulary of $W$ words. Thus, we have two representations for $d$ : (1) $d$, represented by its original terms, and (2) $\hat{d}$, represented by its topic terms. Two corresponding term by document matrices will be denoted by $M_{D}$ and $M_{\hat{D}}$, respectively, where $D$ denotes a set of documents. That is, $M_{D}$ represents the original "text" term by document matrix, while $M_{\hat{D}}$ represents the "topic" term by document matrix.

Figure 1 shows the potential benefit of topic space representation. In the figure, text segments containing target phrase "blow whistle" are projected on a two dimensional subspace. The left figure shows the projection in the "text" space, represented by the term by document matrix $M_{D}$. The middle figure shows the projection in the topic space, represented by $M_{\hat{D}}$. The topic space representation seems to provide a better separation.

We note that when learning topics from a small data sample, learned topics can be less coherent and interpretable, thus less useful. To address this issue, regularized LDA has been proposed in the literature (Newman et al., 2011). A key feature is to favor words that exhibit short range dependencies for a given topic. We can achieve a similar effect by placing restrictions on the vocabulary. For example, when extracting topics from segments containing idioms, we may restrict the vocabulary to contain words from these segments only. The middle and right figures in Figure 1 illustrate a case in point. The middle figure shows a projection onto the topic space that is computed with a restricted vocabulary, while the right figure shows a projection when we place no restriction on the vocabulary. That is, the vocabulary includes terms from documents that contain both idioms and literals.

Note that by computing $M_{\hat{D}}$, the topic term by document matrix, from the training data, we have created a vocabulary, or a set of "features" (i.e., topic terms) that is used to directly describe a query or test segment. The main advantage is that topics are more accurate when computed by LDA from a large collection of idiomatic or literal contexts. Thus, these topics capture more accurately the semantic contexts in which the target idiomatic and literal expressions typically occur. If a target query appears in a similar semantic context, the topics will be able to describe this query as well. On the other hand, one might similarly apply LDA to a given query to extract query topics, and create the query vector from the query topics. The main disadvantage is that LDA may not be able to extract topic terms that match well with those in the training corpus, when applied to the query in isolation.

\subsection{Algorithm}

The main steps of the proposed algorithm, called TopSpace, are shown below.

Input: $D=\left\{d_{1}, \cdots, d_{k}, d_{k+1}, \cdots, d_{n}\right\}:$ training documents of $k$ idioms and $n-k$ literals.

$Q=\left\{q_{1}, \cdots, q_{l}\right\}: l$ query documents.

1. Let DicI be the vocabulary determined solely from idioms $\left\{d_{1}, \cdots, d_{k}\right\}$. Similarly, let $D i c L$ be the vocabulary obtained from literals $\left\{d_{k+1}, \cdots, d_{n}\right\}$.

2. For a document $d_{i}$ in $\left\{d_{1}, \cdots, d_{k}\right\}$, apply LDA to extract a set of $m$ topics $T\left(d_{i}\right)=\left\{t_{1}, \cdots, t_{m}\right\}$ using DicI. For $d_{i} \in\left\{d_{k+1}, \cdots, d_{n}\right\}, D i c L$ is used.

3. Let $\hat{D}=\left\{\hat{d}_{1}, \cdots, \hat{d}_{k}, \hat{d}_{k+1}, \cdots, \hat{d}_{n}\right\}$ be the resulting topic representation of $D$.

4. Compute the term by document matrix $M_{\hat{D}}$ from $\hat{D}$, and let DicT and $g w$ be the resulting dictionary and global weight ( $i d f)$, respectively.

5. Compute the term by document matrix $M_{Q}$ from $Q$, using DicT and $g w$ from the previous step.

Output: $M_{\hat{D}}$ and $M_{Q}$

To summarize, after splitting our corpus (see section 4) into paragraphs and preprocessing it, we extract topics from paragraphs containing idioms and from paragraphs containing literals. We then compute a term by document matrix, where terms are topic terms and documents are topics extracted from the paragraphs. Our test data are represented as a term-by-document matrix as well (See the details in section 5). 

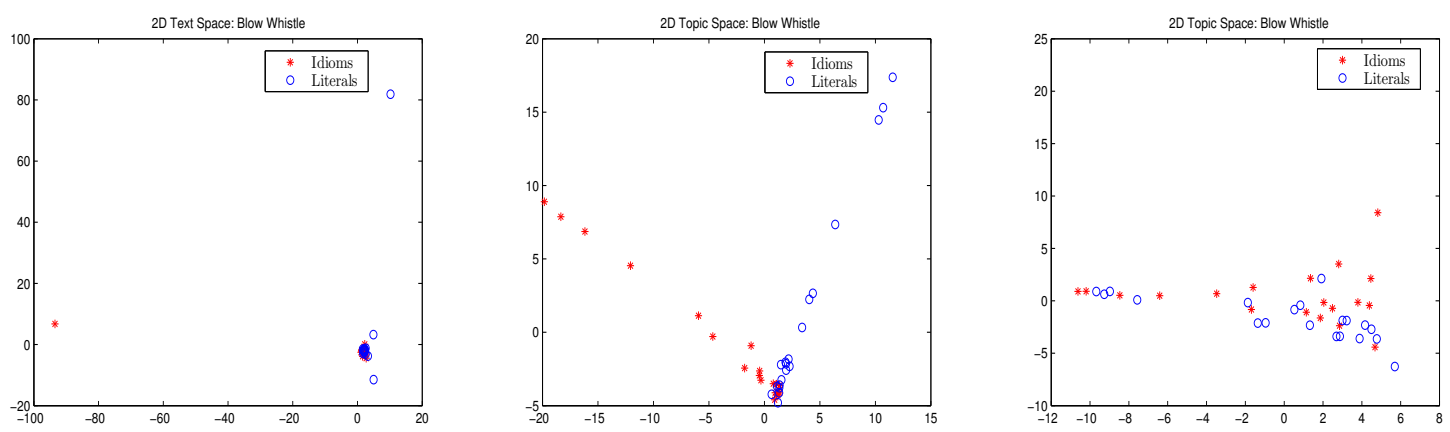

Figure 1: 2D projection of text segments containing “blow whistle.” Left panel: Original text space. Middle panel: Topic space with restricted vocabulary. Right panel: Topic space with enlarged vocabulary.

\subsection{Fisher Linear Discriminant Analysis}

Once $M_{\hat{D}}$ and $M_{Q}$ are obtained, a classification rule can be applied to predict idioms vs. literals. The approach we are taking in this work for classifying idioms vs. literals is based on Fisher's discriminant analysis (FDA) (Fukunaga, 1990). FDA often significantly simplifies tasks such as regression and classification by computing low-dimensional subspaces having statistically uncorrelated or discriminant variables. In language analysis, statistically uncorrelate or discriminant variables are extracted and utilized for description, detection, and classification. Woods et al. (1986), for example, use statistically uncorrelated variables for language test scores. A group of subjects is scored on a battery of language tests, where the subtests measure different abilities such as vocabulary, grammar or reading comprehension. Horvath (1985) analyzes speech samples of Sydney speakers to determine the relative occurrence of five different variants of each of five vowels sounds. Using this data, the speakers cluster according to such factors as gender, age, ethnicity and socio-economic class.

A similar approach has been discussed in Peng et al. (2010). FDA is a class of methods used in machine learning to find the linear combination of features that best separate two classes of events. FDA is closely related to principal component analysis (PCA), where a linear combination of features that best explains the data. Discriminant analysis explicitly exploits class information in the data, while PCA does not.

Idiom classification based on discriminant analysis has several advantages. First, as has been mentioned, it does not make any assumption regarding data distributions. Many statistical detection methods assume a Gaussian distribution of normal data, which is far from reality. Second, by using a few discriminants to describe data, discriminant analysis provides a compact representation of the data, resulting in increased computational efficiency and real time performance.

In FDA, within-class, between-class, and mixture scatter matrices are used to formulate the criteria of class separability. Consider a $J$ class problem, where $m_{0}$ is the mean vector of all data, and $m_{j}$ is the mean vector of $j$ th class data. A within-class scatter matrix characterizes the scatter of samples around their respective class mean vector, and it is expressed by

$$
S_{w}=\sum_{j=1}^{J} p_{j} \sum_{i=1}^{l_{j}}\left(x_{i}^{j}-m_{j}\right)\left(x_{i}^{j}-m_{j}\right)^{t},
$$

where $l_{j}$ is the size of the data in the $j$ th class, $p_{j}$ $\left(\sum_{j} p_{j}=1\right)$ represents the proportion of the $j$ th class contribution, and $t$ denotes the transpose operator. A between-class scatter matrix characterizes the scatter of the class means around the mixture mean $m_{0}$. It is expressed by

$$
S_{b}=\sum_{j=1}^{J} p_{j}\left(m_{j}-m_{0}\right)\left(m_{j}-m_{0}\right)^{t} .
$$

The mixture scatter matrix is the covariance matrix of all samples, regardless of their class assignment, and it is given by

$$
S_{m}=\sum_{i=1}^{l}\left(x_{i}-m_{0}\right)\left(x_{i}-m_{0}\right)^{t}=S_{w}+S_{b} .
$$

The Fisher criterion is used to find a projection matrix $W \in \Re^{q \times d}$ that maximizes

$$
J(W)=\frac{\left|W^{t} S_{b} W\right|}{\left|W^{t} S_{w} W\right|} .
$$

In order to determine the matrix $W$ that maximizes $J(W)$, one can solve the generalized eigenvalue problem: $S_{b} w_{i}=\lambda_{i} S_{w} w_{i}$. The eigenvectors corresponding to the largest eigenvalues form the columns of $W$. For a two class problem, it can be written in a simpler form: $S_{w} w=m=m_{1}-m_{2}$, where $m_{1}$ and $m_{2}$ are the means of the two classes.

\section{Data preprocessing}

\subsection{Verb-noun constructions}

For our experiments we use the British National Corpus (BNC, Burnard (2000)) and a list of verb-noun constructions (VNCs) extracted from BNC by Fazly et al. 
(2009); Cook et al. (2008) and labeled as L (Literal), I (Idioms), or Q (Unknown). The list contains only those VNCs whose frequency was greater than 20 and that occurred at least in one of two idiom dictionaries (Cowie et al., 1983; Seaton and Macaulay, 2002). The dataset consists of 2,984 VNC tokens. For our experiments we only use VNCs that are annotated as I or L.

\subsection{Lemmatization}

Instead of dealing with various forms of the same root, we use lemmas provided by the BNC XML annotation, so our corpus is lemmatized. We also apply the (modified) Google stop list before extracting the topics. The reason we modified the stop list is that some function words can potentially be idiom components (e.g., certain prepositions).

\subsection{Paragraphs}

We use the original SGML annotation to extract paragraghs from BNC. We only kept the paragraphs that contained VNCs for our experiments. We experimented with texts of one paragraph length (single paragraph contexts) and of three-paragraph length (multiparagraph contexts). An example of multi-paragraph contexts is shown below:

So, reluctantly, I joined Jack Hobbs in not rocking the boat, reporting the play and the general uproar with perhaps too much impartiality. My reports went to all British newspapers, with special direct services by me to India, South Africa and West Indies; even to King George V in Buckingham Palace, who loved his cricket. In other words, I was to some extent leading the British public astray.

I regret I can shed little new light on the mystery of who blew the whistle on the celebrated dressing-room scene after Woodfull was hit. while he was lying on the massage table after his innings waiting for a doctor, Warner and Palairet called to express sympathy.

Most versions of Woodfull's reply seem to agree that he said. There are two teams out there on the oval. One is playing cricket, the other is not. This game is too good to be spoilt. It is time some people got out of it. Warner and Palairet were too taken aback to reply. They left the room in embarrassment.

Single paragraph contexts simply consist of the middle paragraph.

\section{Experiments}

\subsection{Methods}

We have carried out an empirical study evaluating the performance of the proposed algorithm. For comparison, the following methods are evaluated. (1) The proposed algorithm TopSpace (1), where the data are represented in topic space. (2) TexSpace algorithm, where the data are represented in original text space. For each representation, two classification schemes are applied: a) FDA (Eq. 4), followed by the nearest neighbor rule. b) SVMs with Gaussian kernels (Cristianini and Shawe-Taylor (2000)). For the nearest neighbor rule, the number of nearest neighbors is set to $\lceil n / 5\rceil$, where $n$ denotes the number of training examples. For SVMs, kernel width and soft margin parameters are set to default values.

\subsection{Data Sets}

The following data sets are used to evaluate the performance of the proposed technique. These data sets have enough examples from both idioms and literals to make our results meaningful. On average, the training data is $6 \mathrm{~K}$ word tokens. Our test data is of a similar size.

BlowWhistle: This data set has 78 examples, 27 of which are idioms and the remaining 51 are literals. The training data for BlowWhistle consist of 40 randomly chosen examples (20 paragraphs containing idioms and 20 paragraphs containing literals). The remaining 38 examples (7 idiomatic and 31 literals) are used as test data.

MakeScene: This data set has 50 examples, 30 of which are paragraphs containing idioms and the remaining 20 are paragraphs containing literals. The training data for MakeScene consist of 30 randomly chosen examples, 15 of which are paragraphs containing make scene as an idiom and the rest 15 are paragraphs containing make scene as a literal. The remaining 20 examples (15 idiomatic paragraphs and 5 literals) are used as test data.

LoseHead: This data set has 40 examples, 21 of which are idioms and the remaining 19 are literals. The training data for LoseHead consist of 30 randomly chosen examples (15 idiomatic and 15 literal). The remaining 10 examples (6 idiomatic and 4 literal) are used as test data.

TakeHeart: This data set has 81 examples, 61 of which are idioms and the remaining 20 are literals. The training data for TakeHeart consist of 30 randomly chosen examples (15 idiomatic and 15 literals). The remaining 51 examples (46 idiomatic and 5 literals) are used as test data.

\subsection{Adding affect}

Nunberg et al. (1994) notice that "idioms are typically used to imply a certain evaluation or affective stance toward the things they denote". Language users usually choose an idiom in non-neutral contexts. The situations that idioms describe can be positive or negative; however, the polarity of the context is not as important as the strength of the emotion expressed. So, we decided to incorporate the knowledge about the emotion strength into our algorithm. We use a database of word norms collected by Warriner et al. (2013). This database contains almost 14,000 English lemmas annotated with three components of emotions: valence (the pleasantness of a stimulus), arousal (the intensity of emotion provoked by a stimulus), and dominance 
Table 1: Average accuracy of competing methods on four datasets in single paragraph contexts: $\mathrm{A}=\mathrm{Arousal}$

\begin{tabular}{|l|ccc|ccc|ccc|ccc|c|c|}
\hline Model & \multicolumn{4}{|c|}{ BlowWhistle } & \multicolumn{4}{c|}{ LoseHead } & \multicolumn{3}{c|}{ MakeScene } & \multicolumn{3}{c|}{ TakeHeart } \\
\hline & Prec & Recall & Acc & Prec & Recall & Acc & Prec & Recall & Acc & Prec & Recall & Acc \\
\hline FDA-Topics & 0.44 & 0.40 & 0.79 & $\mathbf{0 . 7 0}$ & $\mathbf{0 . 9 0}$ & $\mathbf{0 . 7 0}$ & $\mathbf{0 . 8 2}$ & $\mathbf{0 . 9 7}$ & $\mathbf{0 . 8 1}$ & 0.91 & 0.97 & 0.89 \\
FDA-Topics+A & 0.51 & 0.51 & 0.75 & 0.78 & 0.68 & 0.66 & 0.80 & 0.99 & 0.80 & 0.93 & 0.84 & 0.80 \\
FDA-Text & $\mathbf{0 . 3 7}$ & $\mathbf{0 . 8 1}$ & $\mathbf{0 . 6 3}$ & 0.60 & 0.88 & 0.58 & 0.82 & 0.89 & 0.77 & 0.36 & 0.38 & 0.41 \\
FDA-Text+A & 0.42 & 0.49 & 0.76 & 0.64 & 0.92 & 0.63 & $\mathbf{0 . 8 3}$ & $\mathbf{0 . 9 5}$ & $\mathbf{0 . 8 2}$ & 0.75 & 0.53 & 0.53 \\
SVMs-Topics & 0.08 & 0.39 & 0.59 & 0.28 & 0.25 & 0.45 & 0.59 & 0.74 & 0.61 & $\mathbf{0 . 9 1}$ & $\mathbf{1 . 0 0}$ & $\mathbf{0 . 9 1}$ \\
SVMs-Topics+A & 0.06 & 0.21 & 0.69 & 0.38 & 0.18 & 0.44 & 0.53 & 0.40 & 0.44 & $\mathbf{0 . 9 1}$ & $\mathbf{1 . 0 0}$ & $\mathbf{0 . 9 1}$ \\
SVMs-Text & 0.08 & 0.39 & 0.59 & 0.36 & 0.60 & 0.52 & 0.23 & 0.30 & 0.40 & 0.42 & 0.16 & 0.22 \\
SVMs-Text+A & 0.15 & 0.51 & 0.60 & 0.31 & 0.38 & 0.48 & 0.37 & 0.40 & 0.45 & 0.95 & 0.48 & 0.50 \\
\hline
\end{tabular}

(the degree of control exerted by a stimulus). These components were elicited from human subjects via an Amazon Mechanical Turk crowdsourced experiment. We only used the arousal feature in our experiments because we were interested in the intensity of the emotion rather than its valence.

For a document $d=\left\{w_{1}, \cdots, w_{N}\right\}^{t}$, we calculate the corresponding arousal value $a_{i}$ for each $w_{i}$, obtaining $d_{A}=\left\{a_{1}, \cdots, a_{N}\right\}^{t}$. Let $m_{A}$ be the average arousal value calculated over the entire training data. The centered arousal value for a training document is obtained by subtracting $m_{A}$ from $d_{A}$, i.e., $\bar{d}_{A}=$ $d_{A}-m_{A}=\left\{a_{1}-m_{A}, \cdots, a_{N}-m_{A}\right\}^{t}$. Similarly, the centered arousal value for a query is computed according to $\bar{q}_{A}=q_{A}-m_{A}=\left\{q_{1}-m_{A}, \cdots, q_{N}-m_{A}\right\}^{t}$. That is, the training arousal mean is used to center both training and query arousal values. The corresponding arousal matrices for $D, \hat{D}$, and $Q$ are $A_{D}, A_{\hat{D}}, A_{Q}$, respectively. To incorporate the arousal feature, we simply compute

$$
\Theta_{D}=M_{D}+A_{D}
$$

and

$$
\Theta_{\hat{D}}=M_{\hat{D}}+A_{\hat{D}} .
$$

The arousal feature can be similarly incoporated into query $\Theta_{Q}=M_{Q}+A_{Q}$.

\section{Results}

Table 1 shows the average precision, recall, and accuracy of the competing methods on the four data sets over 10 runs in simple paragraph contexts. Table 2 shows the results for the multi-paragraph contexts. Note that for single paragraph contexts, we chose two topics, each having 10 terms. For multi-paragrah contexts, we had four topics, with 10 terms per topic. No optimization was made for selecting the number of topics as well as the number of terms per topic. In the tables, the best performance in terms of the sum of precision, recall and accuracy is given in boldface.

The results show that the topic representation achieved the best performance in 6 out of 8 cases. Figure 2 plots the overall aggregated performance in terms of topic vs text representations across the entire data sets, regardless of the classifiers used. Everything else being equal, this clearly shows the advantage of topics over simple text representation.

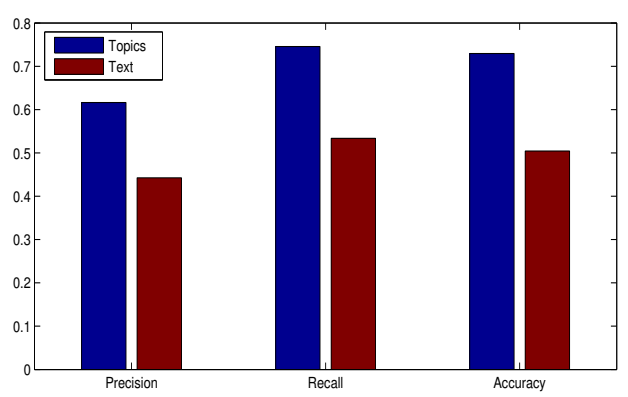

Figure 2: Aggregated performance: Topic vs text representations.

The arousal feature (Eqs 5 and 6) also improved the overall performance, particularly in text representation (Eq. 5). This can be seen in the top panel in Figure 3. In fact, in $2 / 8$ cases, text representation coupled with the arousal feature achieved the best performance. One possible explanation is that the LDA model already performed "feature" selection (choosing topic terms), to the extent possible. Thus, any additional information such as arousal only provides marginal improvement at the best (bottom panel in Figure 3). On the other hand, original text represents "raw" features, whereby arousal information helps provide better contexts, thus improving overall performance.

Figure 4 shows a case in point: the average (sorted) arousal values of idioms and literals of the target phrase "lose head." The upper panel plots arousal values in the text space, while lower panel plots arousal values in the topic space. The plot supports the results shown in Tables 1 and 2, where the arousal feature generally improves text representation.

\section{Comparisons with other approaches}

Even though we used Fazly et al. (2009)'s dataset for these experiments, the direct comparison with their method is impossible here because our task is formulated differently and we do not use the full dataset for the experiments. Fazly et al. (2009)'s unsupervised 
Table 2: Average accuracy of competing methods on four datasets in multiple paragraph contexts: A = Arousal

\begin{tabular}{|l|ccc|ccc|cccc|ccc|}
\hline Model & \multicolumn{4}{|c|}{ BlowWhistle } & \multicolumn{4}{c|}{ LoseHead } & \multicolumn{3}{c|}{ MakeScene } & \multicolumn{3}{c|}{ TakeHeart } \\
\hline & Prec & Recall & Acc & Prec & Recall & Acc & Prec & Recall & Acc & Prec & Recall & Acc \\
\hline FDA-Topics & $\mathbf{0 . 6 2}$ & $\mathbf{0 . 6 0}$ & $\mathbf{0 . 8 3}$ & $\mathbf{0 . 7 6}$ & $\mathbf{0 . 9 7}$ & $\mathbf{0 . 7 8}$ & 0.79 & 0.95 & 0.77 & $\mathbf{0 . 9 3}$ & $\mathbf{0 . 9 9}$ & $\mathbf{0 . 9 2}$ \\
FDA-Topics+A & 0.47 & 0.44 & 0.79 & 0.74 & 0.93 & 0.74 & 0.82 & 0.69 & 0.65 & 0.92 & 0.98 & 0.91 \\
FDA-Text & 0.65 & 0.43 & 0.84 & 0.72 & 0.73 & 0.65 & 0.79 & 0.95 & 0.77 & 0.46 & 0.40 & 0.42 \\
FDA-Text+A & 0.45 & 0.49 & 0.78 & 0.67 & 0.88 & 0.65 & $\mathbf{0 . 8 0}$ & $\mathbf{0 . 9 9}$ & $\mathbf{0 . 8 0}$ & 0.47 & 0.29 & 0.33 \\
SVMs-Topics & 0.07 & 0.40 & 0.56 & 0.60 & 0.83 & 0.61 & 0.46 & 0.57 & 0.55 & 0.90 & 1.00 & 0.90 \\
SVMs-Topics+A & 0.21 & 0.54 & 0.55 & 0.66 & 0.77 & 0.64 & 0.42 & 0.29 & 0.41 & 0.91 & 1.00 & 0.91 \\
SVMs-Text & 0.17 & 0.90 & 0.25 & 0.30 & 0.50 & 0.50 & 0.10 & 0.01 & 0.26 & 0.65 & 0.21 & 0.26 \\
SVMs-Text+A & 0.24 & 0.87 & 0.41 & 0.66 & 0.85 & 0.61 & 0.07 & 0.01 & 0.26 & 0.74 & 0.13 & 0.20 \\
\hline
\end{tabular}
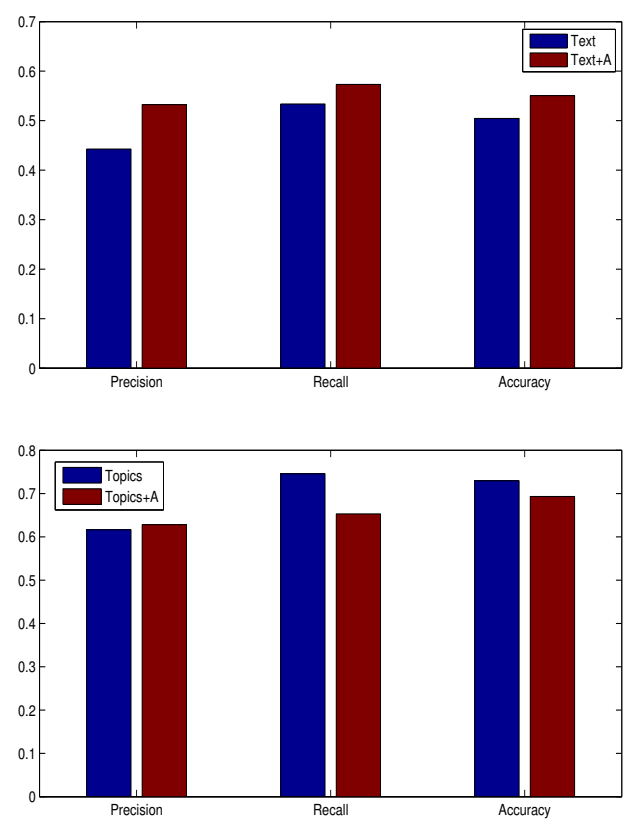

Figure 3: Aggregated performance: Text vs. text+Arousal representations (top) and Topics vs. Topics+Arousal representations (bottom).

model that relies on the so-called canonical forms gives $72.4 \%$ (macro-)accuracy on the extraction of idiomatic tokens when evaluated on their test data.

We cannot compare our method directly with the other methods discussed in section 2 either because each uses a different dataset or formulates the task differently (detection vs. recognition vs. identification). However, we can compare the method presented here with Feldman and Peng (2013) who also experiment with LDA, use similar data, and frame the problem as classification. Their goal, however, is to classify sentences as either idiomatic or literal. To obtain a discriminant subspace, they train their model on a small number of randomly selected idiomatic and nonidiomatic sentences. They then project both the training and the test data on the chosen subspace and use the three nearest neighbor $(3 \mathrm{NN})$ classifier to obtain accuracy. The average accuracy they report is $80 \%$.
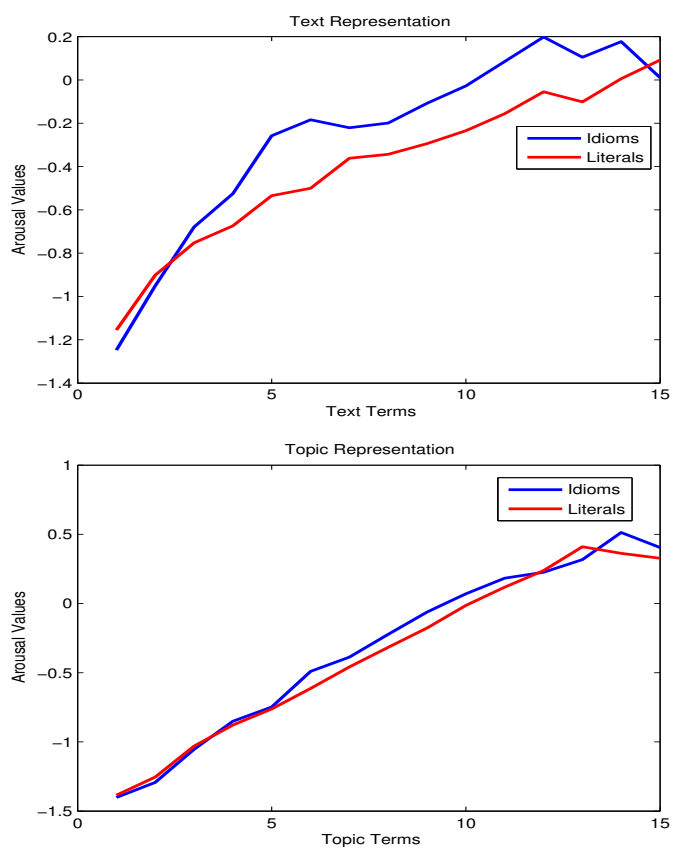

Figure 4: Average arousal values-Upper panel: Text space. Lower panel: Topic space.

Our method clearly outperforms the Feldman and Peng (2013) approach (at least on the dataset we use).

\section{Discussion and Conclusion}

We have described an algorithm for automatic classification of idiomatic and literal expressions. We have investigated the bag of words topic representation for target documents (segments of one or three paragraphs that contains a target phrase). The approach definitely outperforms the baseline model that is based on the simple bag of words representation, but it also outperforms approaches previously discussed in the literature. Our model captures the local semantics and thus is capable to identify semantic outliers (=idioms).

While we realize that the data set we use is small, the results are encouraging. We notice that using 3 paragraphs for local contexts improves the performance of the classifiers. The reason is that some paragraphs are 
relatively short. A larger context provides more related terms, which gives LDA more opportunities to sample these terms.

Idioms are also relatively non-compositional. While we do not measure their non-compositionality in this approach, we indirectly touch upon this property by hypothesizing that non-compositional idiomatic expressions are likely to be far from the local topics.

We feel that incorporating the intensity of emotion expressed by the context into our model improves performance, in particular, in text representation. When we performed a qualitative analysis of the results trying to determine the causes of false positives and negatives, we noticed that there were quite a number of cases that improved after incorporating the arousal feature into the model. For example, the FDA:topic classifier labels "blow the whistle" as literal in the following context, but FDA:topics+A marks this expression as idiomatic (italicized words indicate words with relatively high arousal values):

Peter thought it all out very carefully. He decided the wisest course was to pool all he had made over the last two years, enabling Julian to purchase the lease of a high street property. This would enable them to set up a business on a more settled and permanent trading basis. Before long they opened a grocery-cum-delicatessen in a good position as far as passing trade was concerned. Peter's investment was not misplaced. The business did very well with the two lads greatly appreciated locally for their hard work and quality of service. The range of goods they were able to carry was welcomed in the area, as well as lunchtime sandwich facilities which had previously been missing in the neighbourhood.

Success was the fruit of some three years' strenuous work. But it was more than a shock when Julian admitted to Peter that he had been running up huge debts with their bank. Peter knew that Julian gambled, but he hadn't expected him to gamble to that level, and certainly not to use the shop as security. With continual borrowing over two years, the bank had blown the whistle. Everything was gone. Julian was bankrupt. Even if they'd had a formal partnership, which they didn't, it would have made no difference. Peter lost all he'd made, and with it his chance to help his parents and his younger brother and sister, Toby and Laura.

Peter was heartbroken. His father had said all along: neither a lender nor a borrower. Peter had found out the hard way. But as his mother observed, he was the same Peter, he'd pick himself up somehow. Once again, Peter was resolute. He made up his mind he'd never make the same mistake twice. It wasn't just the money or the hard work, though the waste of that was difficult enough to accept. Peter had been working a debt of love. He'd done all this for his parents, particularly for his father, whose dedication to his children had always impressed Peter and moved him deeply. And now it had all come to nothing.

Therefore, we think that idioms have the tendency to appear in more affective contexts; and we think that incorporating more sophisticated sentiment analysis into our model will improve the results.

\section{Acknowledgments}

This material is based upon work supported by the National Science Foundation under Grant No. 1319846. We also thank the anonymous reviewers for useful comments. The third author thanks the Fulbright Foundation for giving her an opportunity to conduct this research at Montclair State University (MSU).

\section{References}

Ariel, M. (2002). The demise of unique concept of literal meaning. Journal of Pragmatics 34, 361-402.

Birke, J. and A. Sarkar (2006). A clustering approach to the nearly unsupervised recognition of nonliteral language. In Proceedings of the 11th Conference of the European Chapter of the Association for Computational Linguistics (EACL'O6), Trento, Italy, pp. 329-226.

Blei, D., A. Ng, and M. Jordan (2003). Latent Dirichlet Allocation. Journal of Machine Learning Research 3, 993-1022.

Burnard, L. (2000). The British National Corpus Users Reference Guide. Oxford University Computing Services.

Cilibrasi, R. and P. M. B. Vitányi (2007). The google similarity distance. IEEE Trans. Knowl. Data Eng. 19(3), 370-383.

Cook, P., A. Fazly, and S. Stevenson (2007). Pulling their weight: Exploiting syntactic forms for the automatic identification of idiomatic expressions in context. In Proceedings of the ACL 07 Workshop on A Broader Perspective on Multiword Expressions, pp. 41-48.

Cook, P., A. Fazly, and S. Stevenson (2008, June). The VNC-Tokens Dataset. In Proceedings of the LREC Workshop: Towards a Shared Task for Multiword Expressions (MWE 2008), Marrakech, Morocco.

Cowie, A. P., R. Mackin, and I. R. McCaig (1983). Oxford Dictionary of Current Idiomatic English, Volume 2. Oxford University Press.

Cristianini, N. and J. Shawe-Taylor (2000). An Introduction to Support Vector Machines and other kernel-based learning methods. Cambridge, UK: Cambridge University Press.

Fazly, A., P. Cook, and S. Stevenson (2009). Unsupervised Type and Token Identification of Idiomatic Expressions. Computational Linguistics 35(1), 61103.

Feldman, A. and J. Peng (2013). Automatic detection of idiomatic clauses. In Computational Linguistics and Intelligent Text Processing, pp. 435-446. Springer.

Fukunaga, K. (1990). Introduction to statistical pattern recognition. Academic Press.

Hearst, M. A. (1992). Automatic acquisition of hyponyms from large text corpora. In Proceedings of the 14th Conference on Computational Linguistics - Volume 2, COLING '92, Stroudsburg, PA, USA, pp. 539-545. Association for Computational Linguistics. 
Horvath, B. M. (1985). Variation in Australian English. Cambridge: Cambridge University PRess.

Katz, G. and E. Giesbrech (2006). Automatic Identification of Non-compositional Multiword Expressions using Latent Semantic Analysis. In Proceedings of the ACL/COLING-06 Workshop on Multiword Expressions: Identifying and Exploiting Underlying Properties, pp. 12-19.

Li, L. and C. Sporleder (2010). Using Gaussian Mixture Models to Detect Figurative Language in Context. In Proceedings of NAACL/HLT 2010.

Newman, D., E. V. Bonilla, and W. L. Buntine (2011). Improving topic coherence with regularized topic models. In NIPS, pp. 496-504.

Nunberg, G., I. A. Sag, and T. Wasow (1994). Idioms. Language 70(3), 491-538.

Pado, S. and M. Lapata (2007). Dependency-based construction of semantic space models. Computational Linguistics 33(2), 161-199.

Peng, J., A. Feldman, and L. Street (2010). Computing linear discriminants for idiomatic sentence detection. Research in Computing Science, Special issue: Natural Language Processing and its Applications 46, 17-28.

Sag, I. A., T. Baldwin, F. Bond, A. Copestake, and D. Flickinger (2002). Multiword expressions: A Pain in the Neck for NLP. In Proceedings of the 3rd International Conference on Intelligence Text Processing and Computational Linguistics (CICLing 2002), Mexico City, Mexico, pp. 1-15.

Seaton, M. and A. Macaulay (Eds.) (2002). Collins COBUILD Idioms Dictionary (second ed.). HarperCollins Publishers.

Sporleder, C. and L. Li (2009). Unsupervised Recognition of Literal and Non-literal Use of Idiomatic Expressions. In EACL '09: Proceedings of the 12th Conference of the European Chapter of the Association for Computational Linguistics, Morristown, NJ, USA, pp. 754-762. Association for Computational Linguistics.

Warriner, A. B., V. Kuperman, and M. Brysbaert (2013). Norms of valence, arousal, and dominance for 13,915 english lemmas. Behavior Research Methods 44(4).

Widdows, D. and B. Dorow (2005). Automatic extraction of idioms using graph analysis and asymmetric lexicosyntactic patterns. In Proceedings of the ACL-SIGLEX Workshop on Deep Lexical Acquisition, DeepLA '05, Stroudsburg, PA, USA, pp. 4856. Association for Computational Linguistics.

Woods, A., P. Fletcher, and A. Hughes (1986). Statistics in Language Studies. Cambridge: Cambridge University Press. 